\title{
Le financement de l'éducation au Burkina Faso : le défi de la scolarisation primaire universelle
}

Education funding in Burkina Faso. The challenge of universal primary education

La financiación de la educación en Burkina Faso : el reto de la escolarización

Yombo Paul Diabouga et Bagnikoué David Bazongo

\section{(2) OpenEdition}

\section{Journals}

Édition électronique

URL : http://journals.openedition.org/ries/3698

DOI : 10.4000/ries.3698

ISSN : 2261-4265

Éditeur

Centre international d'études pédagogiques

Édition imprimée

Date de publication : 15 avril 2014

Pagination : 111-120

ISBN : 978-2-85420-603-6

ISSN : $1254-4590$

Référence électronique

Yombo Paul Diabouga et Bagnikoué David Bazongo, « Le financement de l'éducation au Burkina Faso : le défı de la scolarisation primaire universelle », Revue internationale d'éducation de Sèvres [En ligne], 65 avril 2014, mis en ligne le 15 avril 2016, consulté le 06 janvier 2020. URL : http:// journals.openedition.org/ries/3698; DOI : 10.4000/ries.3698 


\section{Le financement de l'éducation au Burkina Faso : le défi de la scolarisation primaire universelle}

\section{Yombo Paul Diabouga Bagnikoué David Bazongo}

Lors du sommet du millénaire de l'ONU en 2000, les gouvernements de tous les pays du monde se sont engagés à améliorer le sort du milliard d'êtres humains qui n'a pas assez pour survivre. À cette fin, ils se sont accordés sur huit objectifs à atteindre d'ici 2015 : les Objectifs du millénaire pour le développement (OMD). Parmi ces OMD figurent l'achèvement d'une scolarité primaire de qualité par tous les enfants d'âge scolaire et l'atteinte de la parité filles/ garçons dans l'éducation. Ces objectifs s'inscrivent dans le cadre d'un engagement mondial en faveur de l'Éducation pour tous (EPT), pris lors de la Conférence de l'éducation pour tous tenue à Jomtien (Thaïlande) en 1990 puis réaffirmé à Dakar en 2000, d'offrir une éducation de base de qualité à tous les enfants, jeunes et adultes.

Le Burkina Faso, à l'instar de la plupart des pays du monde, s'est engagé à atteindre l'EPT à l'horizon fixé. À cet effet, plusieurs efforts sont déployés pour l'élaboration et la mise en œuvre de politiques éducatives soutenables. C'est ainsi que l'éducation a occupé une place importante dans les politiques de développement du Burkina Faso depuis 2000. Elle figure en bonne place dans les priorités identifiées dans les documents d'orientation, comme le Cadre stratégique de lutte contre la pauvreté (CSLP) clôturé en 2010 et la Stratégie de croissance accélérée et de développement durable (SCADD) en cours. Cette priorité se traduit également par une allocation conséquente de ressources pour mettre en œuvre les programmes de développement de l'éducation. Aussi, l'étude de l'évolution des dépenses en matière d'éducation au cours des dernières années permet d'apprécier les efforts consentis à tous les niveaux. Quelle est la contribution des différents financeurs au développement de l'éducation ? Comment sont réparties les ressources disponibles entre les différents niveaux du système éducatif ? Cet effort financier produit-il des résultats tangibles? Le présent article vise à apporter des réponses succinctes à ces interrogations. 


\section{LIMITE DU CHAMP DE L'ANALYSE}

Aux termes de la Loi d'orientation de l'éducation adoptée en 2007, l'éducation se définit comme l'ensemble des activités visant à développer chez l'être humain les potentialités physiques, intellectuelles, morales, spirituelles, psychologiques et sociales, en vue d'assurer sa socialisation, son autonomie, son épanouissement et sa participation au développement économique, social et culturel. Elle comprend l'éducation formelle, l'éducation non formelle, l'éducation informelle et l'éducation spécialisée. Dans la présente analyse des coûts et financements de l'éducation, on s'en tiendra à l'éducation formelle et non formelle, les autres domaines étant peu développés ou difficiles à appréhender.

Les dépenses des familles pour l'éducation ne seront pas non plus prises en compte, bien que des enquêtes menées par l'Institut national de la statistique et de la démographie (INSD) et d'autres études montrent que les parents d'élèves supportent certaines dépenses pour l'éducation des enfants. Pour des raisons d'indisponibilité de certaines données relatives aux dépenses des ménages, seul le financement public sera examiné. Il en est de même des dépenses relatives à la formation professionnelle dispensée dans les écoles de formation publiques ou privées, notamment celles qui relèvent du " post-secondaire non supérieur ", niveau 4 de la classification internationale type de l'éducation (CITE). Il s'agit par exemple de l'École nationale d'administration et de magistrature (ENAM), de l'École nationale des douanes, des eaux et forêts, de la police, etc.

\section{ÉVOLUTION DES DÉPENSES PUBLIQUES D'ÉDUCATION}

Bien que l'on entende souvent déclarer que "l'éducation n'a pas de prix " compte tenu des bénéfices qu'elle engendre, force est de reconnaître qu'elle représente une lourde charge pour les États. Au cours de cette dernière décennie, le Burkina Faso a alloué une bonne partie de ses ressources à l'éducation de ses enfants. Pour rapprocher le niveau d'allocation des ressources au profit du ministère de l'éducation nationale et de l'alphabétisation (MENA) et du ministère des enseignements secondaire et supérieur (MESS), on présentera l'évolution récente des dépenses dans le tableau 1.

De 2008 à 2012, le budget de l'État a connu un accroissement global de $163,42 \%$, alors que celui de l'enseignement de base a augmenté de $157,2 \%$ et celui des enseignements secondaire et supérieur de 177,4\%. Cela signifie-t-il que le pays accorde la priorité à son système éducatif ? En répondant à cette interrogation sur la base du volume de ressources consacrées à l'éducation, on peut facilement se tromper. Il est plus judicieux de mener l'analyse sur la base des critères internationaux.

L'initiative de mise en œuvre accélérée (IMOA) de l'Éducation pour nous (EPT) connue sous l'appellation de Fast Track Initiative (FTI) est un 
Tableau 1. Évolution des budgets du MENA et du MESS 2008-2012 en milliers de francs CFA ${ }^{1}$

\begin{tabular}{|l|c|c|c|}
\hline \multicolumn{1}{|c|}{ Années } & $\mathbf{2 0 0 8}$ & $\mathbf{2 0 1 2}$ & $\begin{array}{c}\text { Variation annuelle } \\
\text { moyenne (\%) }\end{array}$ \\
\hline Budget MENA & 81926022 & 128761973 & 11,97 \\
\hline Dont CAST ${ }^{2}$ & 23848000 & 15482000 & 15,41 \\
\hline Budget MESS & 44345274 & 78682418 & 13,21 \\
\hline Budget MENA+MESS & 126295144 & 207459873 & 13,06 \\
\hline Budget total éducation & 694804215 & 1135428542 & 0,13 \\
\hline $\begin{array}{l}\text { Éducation/ } \\
\text { Total du budget (\%) }\end{array}$ & $18,18 \%$ & $18,27 \%$ & \\
\hline
\end{tabular}

Source : Lois de règlement et IAP, avril 2013.

partenariat mondial évolutif, créé en 2002 à la suite du consensus sur le développement réalisé à la conférence de Monterrey. Elle est ouverte aux pays à faible revenu qui peuvent y adhérer après examen des plans de développement des systèmes éducatifs. Après plusieurs études dans le cadre de cette initiative, on est parvenu à la conclusion que l'ensemble des pays qui avaient réussi à développer véritablement leur système éducatif présentaient des traits communs. On notait en particulier qu'ils consacraient au moins $20 \%$ de leurs ressources budgétaires à l'éducation et que 42 à $64 \%$ de ces ressources étaient consacrées à l'enseignement primaire. On peut considérer que ces données constituent de bons indicateurs quant à la priorité à accorder au système éducatif. Ils constituent, avec d'autres, ce qui est appelé le « cadre indicatif du FTI».

En partant de ces éléments d'analyse, on remarque que le Burkina Faso a certes fait des efforts en termes d'allocation de ressources mais que la part consacrée à l'éducation est toujours en deçà des objectifs du cadre indicatif. Il est donc important que la programmation au niveau national tienne compte de ces éléments objectifs pour permettre un développement conséquent du système éducatif.

\section{LES FINANCEMENTS SUR RESSOURCES EXTÉRIEURES}

L'éducation burkinabè bénéficie d'appuis extérieurs et l'analyse ci-après permet de se faire une idée globale des interventions des partenaires techniques et financiers.

\footnotetext{
1. 1 euro $=655,589$ francs CFA.

2. CAST : Compte d'affectation spéciale du Trésor, qui retrace les aides budgétaires sectorielles des donneurs internationaux.
} 
Six partenaires interviennent actuellement sous forme d'aide budgétaire sectorielle par le biais d'un fonds commun géré sous la forme d'un compte d'affectation spéciale du Trésor (CAST). Ces partenaires sont: l'Agence française de développement (AFD), le Canada, le Danemark, la Suisse, l'Unicef, le Luxembourg. La Délégation de l'Union européenne n'alimente plus le CAST depuis 2007 et intervient dorénavant sous une autre forme appelée "aide budgétaire générale ».

Outre ces partenaires, le Burkina Faso a bénéficié, au cours de la décennie, d'autres types de fonds qui sont gérés dans le CAST : les fonds catalytiques et les fonds du Partenariat mondial pour l'éducation (PME).

Les fonds catalytiques sont des fonds mis en place par certains bailleurs de fonds, qui viennent en aide aux systèmes éducatifs crédibles dont les ressources apportées par les partenaires ne couvrent pas les besoins. En 2003, le Burkina Faso, après son adhésion à l'initiative Fast Track, a introduit une requête pour ce type de financement. Avec le nombre de donateurs relativement élevé dans le secteur de l'éducation, le pays n'a pas été retenu alors pour un financement par le fonds catalytique $(\mathrm{CF})$ là sur la base du critère "d'orphelin de donateurs ".

Une deuxième requête de 2008 a permis au pays de bénéficier d'un appui de 51 milliards de francs CFA. La Banque mondiale a été l'agence de supervision de ce financement FTI.

En mars 2013, une autre requête a permis d'obtenir la somme de 78,2 millions de dollars US, soit un peu plus de 39 milliards de francs CFA, accordé au Burkina Faso par le PME sur la période 2014-2016. L'Agence française de développement est l'agence de supervision de ce fonds, dont la première tranche d'un montant de 14 milliards de francs CFA sera débloquée courant mars 2014.

Le tableau 2 donne la part des différents partenaires dans le CAST, sur les quatre dernières années. Ainsi, entre 2011 et 2014, l'éducation de base a bénéficié des partenaires intervenant dans le CAST d'un montant de 73,3 milliards FCFA.

En dehors du CAST, d'autres partenaires importants, bailleurs de fonds et ONG, interviennent sur tout le secteur de l'éducation de base, directement dans les localités ou sous forme de projets. Ainsi, au titre de l'année 2013, on a répertorié les interventions au profit de l'éducation de base sous forme de projets dans le tableau 3.

Ce tableau montre que les projets intervenant dans l'éducation apportent une part significative à l'éducation de base. Le montant cumulé pour l'année 2013 est de 16 milliards de francs CFA. Pour 2014, les prévisions se chiffrent à 13,339 milliards de francs CFA.

Les mécanismes actuels de collecte de données ne permettent pas de fournir une situation exhaustive de la contribution à l'éducation des autres acteurs, notamment des ONG et des associations, ainsi que les financements sur ressources propres des communes et les financements privés. Toutefois, on peut 
Tableau 2. Contributions des bailleurs au CAST de 2011 à 2014 (en milliers de francs CFA)

\begin{tabular}{|c|c|c|c|c|c|c|c|c|c|}
\hline Libellés & 2011 & $\begin{array}{l}\text { Part } \\
\text { en } \%\end{array}$ & 2012 & $\begin{array}{l}\text { Part } \\
\text { en } \%\end{array}$ & 2013 & $\begin{array}{l}\text { Part } \\
\text { en } \%\end{array}$ & $\begin{array}{c}\text { Montants } \\
\text { prévus } 2014\end{array}$ & $\begin{array}{l}\text { Part } \\
\text { en } \%\end{array}$ & $\begin{array}{c}\text { Total } \\
\text { 2011-2014 }\end{array}$ \\
\hline AFD & 2041000 & 12,27 & 1525100 & 9,85 & 2033467 & 11,66 & 2049866 & 8,63 & 7649433 \\
\hline Suisse & 186496 & 1,12 & 271390 & 1,75 & 2415000 & 13,84 & 263500 & 1,11 & 3136386 \\
\hline Canada & 5397435 & 32,46 & 5287650 & 34,15 & 5623092 & 32,24 & 1616800 & 6,81 & 17924977 \\
\hline Unicef & 770976 & 4,64 & 250000 & 1,61 & 246000 & 1,41 & 250000 & 1,05 & 1516976 \\
\hline Danemark & 1606768 & 9,66 & 1238938 & 8,00 & 1119956 & 6,42 & 877763 & 3,70 & 4843425 \\
\hline Pays-Bas & 4400000 & 26,46 & $3800000^{3}$ & 24,54 & 3800000 & 21,78 & & & 12000000 \\
\hline Luxembourg & & & & & & & 4690093 & 19,75 & 4690093 \\
\hline PME & & & & & & & 14000000 & 58,95 & 14000000 \\
\hline $\begin{array}{l}\text { Report année } \\
\text { antérieure }\end{array}$ & 2224619 & 13,38 & 3109219 & 20,08 & 2206068 & 12,65 & & & 7539906 \\
\hline Total & 16627294 & 100 & 15482297 & 100 & 17443583 & 100 & 23748021 & 100 & 73301196 \\
\hline
\end{tabular}

Sources : Plan d'actions 2012, 2013 et 2014 du MENA.

Tableau 3. Contribution des projets en 2013 (en milliers francs CFA)

\begin{tabular}{|l|c|c|c|}
\hline \multirow{2}{*}{ Intitulé du projet } & \multicolumn{2}{c|}{ Programmation révisée en 2013 } \\
\cline { 2 - 4 } & $\begin{array}{c}\text { Contrepartie } \\
\text { nationale }\end{array}$ & $\begin{array}{c}\text { Ressources } \\
\text { extérieures }\end{array}$ & Total \\
\hline BKF 014 & & 49314 & 49314 \\
\hline ES-CEBNF & 726204 & 3287039 & 4013243 \\
\hline PACOGES & 21287 & 347720 & 369007 \\
\hline Construction d'écoles primaires phase 5 & 37500 & 3797841 & 3835341 \\
\hline Programme de Pays du PAM & 77368 & 1564790 & 1642159 \\
\hline Projet « Une lampe pour l'Afrique » & 167110 & 983936 & 1151046 \\
\hline Cantines scolaires/Cathwel & 81456 & 2119682 & 2301138 \\
\hline BID IV & 197941 & 2007973 & 2205914 \\
\hline SMASE & 194963 & 376732 & 571695 \\
\hline TOTAL & $\mathbf{1 6 0 3 8 2 9}$ & $\mathbf{1 4 5 3 5 0 2 7}$ & $\mathbf{1 6 1 3 8 \mathbf { 8 5 7 }}$ \\
\hline
\end{tabular}

Source : plan d'action 2013 du MENA.

3. Les Pays-Bas via l'Unicef. 
dire, sur la base d'études antérieures, que leur part est significative dans le financement de l'éducation. Un mécanisme est mis en place et permettra prochainement de comptabiliser l'ensemble de ces interventions pour mieux appréhender le montant global des investissements en éducation au Burkina Faso.

\section{Plans de développement DU SYSTÈME ÉDUCATIF BURKINABÈ}

Il y a encore quelques années, il n'était pas rare de voir affiché dans les ministères de l'éducation nationale de certains pays le slogan « si tu trouves que l'éducation coûte cher, essaye l'ignorance ». Cela traduit l'idée que l'on se faisait alors du financement de l'éducation. La planification de l'éducation était alors peu rigoureuse, les plans de développement n'apparaissaient pas nécessaires puisque l'on considérait que cette dernière n'avait pas de prix. Des études comparatives ont permis de se rendre compte que certains pays réussissaient à développer leur système éducatif avec moins de ressources que d'autres. On en a conclu que ce développement n'est pas conditionné à la seule mobilisation de ressources et que la planification et la bonne gestion financière du secteur éducatif en constituent une condition de réussite.

Depuis le début des années 2000, le système éducatif du Burkina Faso s'est engagé dans un processus de gestion axée sur les résultats (GAR), avec des documents de planification stratégique et opérationnelle qui permettent de mieux gérer les ressources disponibles. Les principaux instruments de planification stratégiques sont :

- un plan décennal de développement de l'éducation de base (PDDEB) élaboré en 2000 pour couvrir essentiellement le primaire mais qui a intégré, dans sa deuxième phase, le post-primaire et le préscolaire ;

- un plan de développement stratégique (PDSEB) qui a pris le relais du PDDEB pour la période 2012-2021;

- une politique sous-sectorielle des enseignements secondaire, supérieur et de la recherche scientifique.

Ces documents sous-sectoriels ont été insérés dans des documents de planification sectorielle, le programme sectoriel de l'éducation et de la formation, élaboré en 2012 et révisé en 2014, pour devenir la Politique sectorielle de l'éducation.

\section{RÉPARTITIONS DES RESSOURCES ENTRE LES SOUS-SECTEURS}

La répartition de l'enveloppe budgétaire entre les niveaux du système éducatif fait l'objet de discussions. Au cours des dernières années, l'enseignement primaire a reçu plus de la moitié des ressources consacrées à l'éducation. Il serait tout à fait abusif de croire que le fait d'accorder cette priorité à l'éducation de base relève de la volonté de maintenir une bonne partie de 
la population dans le sous-secteur de l'éducation de base et d'empêcher la formation de l'élite de demain. Cette situation doit être plutôt perçue comme un choix opéré par le pays de développer son système éducatif de manière progressive et ce, en commençant par le primaire pour l'étendre ensuite aux autres niveaux. L'extension de la gratuité et de l'obligation scolaire jusqu'à 16 ans pour prendre en compte le post-primaire, exprimée dans la loi d'orientation de l'éducation de 2007, participe de cette volonté.

Dans les années à venir, les investissements au profit du post-primaire seront accrus pour concrétiser cette vision. Cependant, cela ne signifie pas que les autres niveaux doivent être oubliés car chaque niveau a son importance et le développement du supérieur influence le développement des autres niveaux. On sait par exemple que plus l'enseignement supérieur alimente le marché de l'emploi de sortants qualifiés, plus les autres niveaux bénéficient d'un encadrement de qualité. Il en découle que l'État doit accorder au supérieur toute sa place; l'adoption du plan national d'action pour le développement de l'enseignement supérieur (PNADES) 2014-2023 entre dans ce cadre.

\section{NATURE DES DÉPENSES}

Lorsque l'on examine les dépenses opérées dans le domaine de l'éducation, on s'aperçoit qu'en réalité, elles sont orientées vers deux grands axes : les dépenses de personnel et les constructions d'infrastructures. Le tableau ci-dessous donne l'évolution des budgets en fonction du type de dépenses.

Tableau 4. Répartition des dépenses du MENA et du MESS par titre budgétaire (en \%)

\begin{tabular}{|c|c|c|c|c|c|}
\hline Ministères & Titre $^{4}$ & 2004 & 2009 & 2010 & 2011 \\
\hline \multirow{5}{*}{ MENA } & 2 Personnel & 75 & 74 & 67 & 81 \\
\hline & 3 Fonctionnement hors personnel & 8 & 8 & 6 & 5 \\
\hline & 4 Transferts & 8 & 8 & 6 & 6 \\
\hline & 5 Investissements & 9 & 9 & 21 & 8 \\
\hline & Sous total & 100 & 100 & 100 & 100 \\
\hline \multirow{5}{*}{ MESS } & 2 Personnel & 37 & 36 & 33 & 37 \\
\hline & 3 Fonctionnement hors personnel & 5 & 3 & 2 & 2 \\
\hline & 4 Transferts & 49 & 53 & 52 & 53 \\
\hline & 5 Investissements & 9 & 8 & 12 & 8 \\
\hline & Sous total & 100 & 100 & 100 & 100 \\
\hline
\end{tabular}

Source : Annuaire budgétaire 2011.

4. Selon la nomenclature budgétaire, les dépenses du titre 2 sont celles destinées à la prise en charge des dépenses du personnel, le titre 3 regroupe les dépenses pour l'acquisition de biens et de services pour le fonctionnement courant des services, le titre 4 regroupe les dépenses de transfert courant essentiellement en direction des établissements à autonomie budgétaire (écoles de formation d'enseignants, universités...) et le titre 5 concerne les investissements. 
Le tableau montre que les dépenses de personnel occupent la part la plus importante du budget consacré à l'éducation. Au MENA par exemple, la proportion du budget relatif aux dépenses de personnel ${ }^{5}$ a toujours dépassé $65 \%$ sur la période. En effet, il est indéniable que l'enseignant est l'élément central du système éducatif. L'ensemble des études dans le domaine de l'éducation ont montré que pour accroître l'accès à l'éducation, il ne suffit pas de construire des salles de classes mais il faut aussi et surtout s'assurer de la disponibilité du personnel enseignant. En matière de qualité également, la place de l'enseignant n'est plus à démontrer. Les conditions de travail, la disponibilité des matériels didactiques influencent la réussite des élèves mais ces facteurs ont moins d'impact que la disponibilité de l'enseignant lui-même.

Après les salaires viennent les constructions, qui absorbent une proportion non négligeable des ressources destinées à l'éducation, surtout au niveau du primaire.

\section{LeS RÉsultats de la politique ÉDUCATIVE}

Aborder le financement d'un système éducatif sans regarder les résultats obtenus serait faire preuve d'un manque de réalisme. Les ressources sont allouées dans le but de produire des résultats. Cette conception inspirée de la gestion axée sur les résultats est de plus en plus répandue dans l'éducation.

On peut tout d'abord s'intéresser à des résultats quantitatifs en observant quelques indicateurs d'accès à l'éducation et de couverture. L'effectif des élèves nouvellement inscrits au CP1 a presque triplé entre les années scolaires 2000/2001 et 2012/2013. En effet, le nombre de nouveaux inscrits en première année du primaire est passé de 172162 en 2000/01 à 499401 élèves à 2012/2013, soit un accroissement annuel moyen de 9,3\%. Cette importante hausse des effectifs a ainsi permis d'obtenir des taux brut d'admission (TBA) satisfaisants. Au cours de la même période, l'on a enregistré un TBA de 92,8 \% en 2012 contre 47,4\% en 2000.

$\mathrm{Au}$ post-primaire (premier cycle du secondaire) et au secondaire, les taux bruts d'admission sont respectivement de 38,6 \% et de $16,59 \%$ en 2012 2013. Ceci signifie qu'un peu plus de 38 enfants sur 100 en âge d'être scolarisés en $6^{\mathrm{e}}$ ont pu y avoir accès et que sur 100 enfants en âge d'être scolarisés en première année du secondaire, 17 ont pu y accéder effectivement en 2012/2013.

En ce qui concerne l'enseignement supérieur, le nombre d'étudiants pour 100000 habitants était de 424 en 2011/2012, un indicateur en hausse de 36 points par rapport à l'année $2011 / 2012$ et de 180 points par rapport à l'année $2007 / 2008$.

5. Une partie des dépenses de transfert au niveau du MENA peuvent également être comptabilisées comme des dépenses du personnel car étant destinées au fonctionnement des écoles nationales des enseignants du primaire. 
On peut aussi apprécier les résultats de manière plus qualitative, en tentant de mesurer les performances du système éducatif en termes d'apprentissage des élèves. C'est l'objectif que se sont fixé un ensemble de pays d'Afrique sub-saharienne en lançant, au début des années 1990, le PASEC, programme d'analyse des systèmes éducatifs de la Confemen ${ }^{6}$. Ce programme vise à "mettre en relation les aspects quantitatifs et qualitatifs des systèmes éducatifs et de dégager les choix possibles des stratégies éducatives les plus efficaces. » Il permet d'analyser les performances des systèmes éducatifs, d'en observer l'évolution et de les comparer entre pays culturellement et géographiquement proches. Il s'appuie sur des tests standardisés portant sur le français et les mathématiques administrés dans tous les pays participants, en début $\left(2^{\mathrm{e}}\right.$ année) et en fin du cycle primaire $\left(5^{\mathrm{e}}\right)$ à un large échantillon d'élèves. Les données recueillies permettent, certes, comme les résultats de l'enquête PISA pour les pays de l'OCDE, de comparer les performances des élèves entre les pays et d'observer leur évolution pays par pays, mais leur apport le plus précieux pour la définition des politiques éducatives nationales est l'analyse des facteurs de la performance. L'étude des variations des résultats aux tests en fonction des caractéristiques des élèves et de leurs conditions de scolarisation permet d'estimer quels sont les éléments qui contribuent à l'amélioration des performances et quels sont ceux qui, au contraire, ont un effet négatif.

Que nous apprennent les résultats des enquêtes PASEC dans le cas du Burkina Faso ?7 Tout d'abord, entre les deux enquêtes de 1996 et 2007, les performances des élèves ont chuté, tant en français qu'en mathématiques, aussi bien en début qu'en fin de cycle, notamment en ce qui concerne les élèves des zones rurales. Ceci s'explique par le fait que la croissance rapide de la scolarisation a surtout concerné ces derniers, parmi lesquels ceux issus des familles les plus démunies ont enregistré des résultats les plus faibles.

Tableau 5. Indicateurs du PASEC

\begin{tabular}{|l|c|c|c|c|c|}
\hline & $\begin{array}{c}\text { PIB } \\
\text { par tête } \\
\text { (USD) }\end{array}$ & $\begin{array}{c}\text { Dépenses } \\
\text { publiques par élève } \\
\text { (\% du PIB par tête) }\end{array}$ & $\begin{array}{c}\text { Taux } \\
\mathbf{d} \text { accès } \\
\text { en } \\
\mathbf{5}^{\mathbf{e}} \text { année }\end{array}$ & $\begin{array}{c}\text { Score moyen } \\
\mathbf{5}^{\mathbf{e}} \text { année } \\
\text { en français } \\
\text { (sur 100) }\end{array}$ & $\begin{array}{c}\text { Score moyen } \\
\mathbf{5}^{\mathbf{e}} \text { année } \\
\text { en français } \\
\text { (sur 100) }\end{array}$ \\
\hline Tchad (2004) & 262 & 6,3 & 48 & 34,3 & 34,0 \\
\hline Bénin (2005) & 321 & 11,8 & 57 & 32,1 & 31,9 \\
\hline Madagascar (2005) & 233 & 5,1 & 57 & 39,4 & 52,0 \\
\hline Burkina (2007) & 260 & 16,6 & 42 & 40,1 & 38,2 \\
\hline
\end{tabular}

Source : Étude PASEC Burkina Faso.

6. La Confemen fut créée en 1960 en tant que Conférence des ministres de l'éducation des pays africains et malgache d'expression française.

7. Étude PASEC Burkina Faso (2009). 
En comparaison avec les pays à niveau de revenu moyen comparable, le Burkina Faso dépense beaucoup plus par élève mais enregistre le plus faible taux de rétention des élèves dans le primaire. Ses résultats aux scores de fin de $5^{\mathrm{e}}$ année sont nettement plus élevés que ceux du Tchad et du Bénin et au même niveau que Madagascar en mathématiques, mais inférieurs en français.

Quant aux éléments qui influent sur la réussite, il apparaît que le temps de trajet pour se rendre à l'école et le travail des élèves, dans les champs pour les ruraux ou à domicile, sont des facteurs négatifs très importants. Doublé d'un fort absentéisme des maîtres, qui a aussi un impact négatif, il a pour effet de réduire le temps scolaire effectif.

La disponibilité de manuels scolaires et d'équipement pédagogique joue également un rôle sur les résultats, en particulier en début de cycle, alors que l'ancienneté ou le niveau de formation des maîtres a peu d'impact.

Ceci peut se traduire en priorités dans l'affectation des ressources, comme par exemple l'augmentation des crédits de fonctionnement hors salaires (notamment par une réduction des effectifs des personnels administratifs et techniques) pour permettre d'accroître les dépenses d'équipement et de matériel pédagogique.

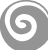

À deux années de l'échéance de 2015, on peut dire que les progrès du Burkina Faso en matière de développement de l'éducation sont très significatifs depuis les années 2000 mais que, si les objectifs de mixité sont près d'être réalisés, les objectifs de scolarisation sont loin d'être atteints. La priorité donnée à l'éducation dans le budget de l'État et la mobilisation de l'aide internationale ne permettront d'atteindre la scolarisation universelle au primaire, de développer le secondaire et l'enseignement supérieur, tout en améliorant la qualité des apprentissages des élèves, qu'au prix d'une affectation rigoureuse des ressources, fondée sur des priorités définies par une efficacité démontrée des différentes modalités de l'action publique.

\section{BibliographiE}

Ministère de l'enseignement de base et de l'alphabétisation et Confemen.

Étude PASEC Burkina Faso «les apprentissages scolaires au Burkina Faso: les effets du contexte, les facteurs pour agir", septembre 2009.

PILON Marc \& WAYACK Madeleine: "La démocratisation de l'enseignement au Burkina Faso : que peut-on en dire aujourd'hui ? ", Cahiers d'études africaines, http :// etudesafricaines.revues.org/190 [consulté le 16 mars 2014].

Plan décennal de développement de l'éducation de base 2000-2009.

Programme de développement stratégique du secteur de l'éducation de base 2012-2021.

Loi $\mathrm{n}^{\circ} 103-2007 / \mathrm{AN}$ du 30 juillet 2007 portant loi d'orientation de l'éducation.

Stratégie de croissance accélérée et de développement durable.

Plans d'action 2012, 2013 et 2014 du MENA. 